\title{
Autophagy in human type 2 diabetes pancreatic beta cells
}

\author{
M. Masini • M. Bugliani • R. Lupi • S. del Guerra • \\ U. Boggi • F. Filipponi • L. Marselli • P. Masiello • \\ P. Marchetti
}

Received: 5 January 2009 / Accepted: 6 March 2009/Published online: 15 April 2009

(C) Springer-Verlag 2009

\begin{abstract}
Aims/hypothesis Beta cell loss contributes to type 2 diabetes, with increased apoptosis representing an underlying mechanism. Autophagy, i.e. the physiological degradation of damaged organelles and proteins, may, if altered, be associated with a distinct form of cell death. We studied several features of autophagy in beta cells from type 2 diabetic patients and assessed the role of metabolic perturbation and pharmacological intervention.

Methods Pancreatic samples were obtained from organ donors and isolated islets prepared both by collagenase digestion and density gradient centrifugation. Beta cell morphology and morphometry were studied by electron microscopy. Gene expression studies were performed by quantitative RT-PCR.

Results Using electron microscopy, we observed more dead beta cells in diabetic $(2.24 \pm 0.53 \%)$ than control $(0.66 \pm$ $0.52 \%)$ samples $(p<0.01)$. Massive vacuole overload (suggesting altered autophagy) was associated with $1.18 \pm$ $0.54 \%$ dead beta cells in type 2 diabetic samples and with
\end{abstract}

M. Masini · P. Masiello

Department of Experimental Pathology, University of Pisa,

Pisa, Italy

M. Bugliani • R. Lupi $\cdot$ S. del Guerra $\cdot$ L. Marselli

P. Marchetti $(\square)$

Department of Endocrinology and Metabolism,

Ospedale Cisanello, via Paradisa 2, 56100 Pisa, Italy

e-mail: marchant@immr.med.unipi.it

\section{U. Boggi}

Department of Oncology, Transplants and Advanced Technologies in Medicine, University of Pisa,

Pisa, Italy

F. Filipponi

Department of Liver Transplant, University of Pisa,

Pisa, Italy
$0.36 \pm 0.26 \%$ in control samples $(p<0.05)$. Density volume of autophagic vacuoles and autophagosomes was significantly higher in diabetic beta cells. Unchanged gene expression of beclin-1 and $A T G 1$ (also known as ULK1), and reduced transcription of $L A M P 2$ and cathepsin $\mathrm{B}$ and $\mathrm{D}$ was observed in type 2 diabetic islets. Exposure of nondiabetic islets to increased NEFA concentration led to a marked increase of vacuole accumulation, together with enhanced beta cell death, which was associated with decreased LAMP2 expression. Metformin ameliorated autophagy alterations in diabetic beta cells and beta cells exposed to NEFA, a process associated with normalisation of $L A M P 2$ expression.

Conclusions/interpretation Beta cells in human type 2 diabetes have signs of altered autophagy, which may contribute to loss of beta cell mass. To preserve beta cell mass in diabetic patients, it may be necessary to target multiple cell-death pathways.

Keywords Autophagy - Metformin · NEFA ·

Pancreatic beta cells

\author{
Abbreviations \\ AMPK AMP kinase \\ ATG Autophagy-related gene \\ LAMP Lysosome-associated membrane protein \\ mTOR Mammalian target of rapamycin (mTOR)
}

\section{Introduction}

In type 2 diabetes, increased apoptosis, a type of programmed cell death, contributes to beta cell loss [1]. However, additional models of cell death have been 
described [2]. One involves macroautophagy (referred to hereafter as autophagy), which is characterised by absence of chromatin condensation and massive cytoplasmic vacuolisation. Autophagy is a self-digesting mechanism responsible for removal of organelles and defined cytoplasmic regions. It begins when flat membrane cisterns wrap themselves around organelles with or without portions of cytosol, forming closed double membrane vacuoles (autophagic vacuoles). These structures then mature in stages, involving fusion events with primary lysosomes (autophagosomes or autolysosomes), which deliver the hydrolytic enzymes responsible for degradation of the engulfed material. Normally, autophagy is beneficial, since it regulates aged protein turnover, eliminates damaged organelles and protects cells during starvation [3]. However, conspicuous autophagy may trigger the cell to die in a nonapoptotic manner. In fact, altered autophagy may be implicated in diseases such as cancer, neurodegenerative disorders and hepatic encephalopathy [4]; potential roles in diabetes have also been suggested. Thus accumulation of autophagic vacuoles was observed in interleukin-1 exposed beta cells [5]. More recently, altered autophagy was observed in beta cells of Zucker diabetic fatty rats and in a beta cell line following high glucose exposure [6]. Furthermore, unbalanced degradative pathways contributed to insulin secretion deficiency in $R a b 3 a^{-/-}$mice, with intracellular vacuoles accumulation [7]. Finally, autophagosome accumulation was reported in beta cells of diabetic $d b / d b$ and in nondiabetic high-fat-fed C57BL/6 mice, while beta cell-specific deletion of autophagy-related gene (ATG) 7 , a molecule involved in autophagy, was shown to cause beta cell dysfunction in mice $[3,8]$. Therefore, we investigated whether altered autophagy may contribute to beta cell damage in human type 2 diabetes.

\section{Methods}

Pancreas donors Pancreatic samples and/or isolated islets of 26 multiorgan donors (14 with type 2 diabetes: age, $66.5 \pm 8.2$ years; BMI, $27.1 \pm 3.6 \mathrm{~kg} / \mathrm{m}^{2} ; 12$ non-diabetic controls: age, $64 \pm 13$ years; BMI, $25.5 \pm 2.3 \mathrm{~kg} / \mathrm{m}^{2}$ ) were studied, with the approval of our local ethics committee. In the diabetic group, one patient had been treated by diet alone, three with metformin, three with sulfonylureas, three with combined sulfonylurea and metformin, and two with insulin therapy. Causes of death were similar in both donor groups (trauma [two in each group], cardiovascular events [12 and ten in diabetic and control respectively]). Cold ischaemia time was $<14 \mathrm{~h}$ in both groups.

Electron microscopy evaluation Electron microscopy studies were performed on pancreatic samples and isolated islets as previously described [9]. Cells were considered dead on the basis of any of the following criteria: loss of plasma membrane integrity, fragmentation into discrete bodies, engulfment of cell corpse or its fragments by an adjacent cell [2]. The presence of marked chromatin condensation and/or blebs was considered to be a sign of apoptosis, whereas massive vacuole accumulation and absence of chromatin condensation were treated as signs of cell death associated with autophagy [2]. Autophagic vacuoles were identified from the presence of organelles and/or cytoplasmic portions surrounded by close double membranes; autophagosomes were identified from the presence of single membrane vacuoles containing organelles with signs of degradation $[2,4]$. For morphometric studies, volume density values were derived as described [9].

Islet isolation and culture Islets were isolated by enzymatic digestion and gradient purification, and then cultured in M199 medium at $5.5 \mathrm{mmol} / \mathrm{l}$ glucose until experiments were performed $[9,10]$. In some cases, islets from nondiabetic donors were evaluated after $24 \mathrm{~h}$ incubation at $1.0 \mathrm{mmol} / \mathrm{l} \mathrm{NEFA} \mathrm{(oleate/palmitate,} \mathrm{2:1)} \mathrm{[10].} \mathrm{The} \mathrm{effects}$ of $24 \mathrm{~h}$ culture with $2.4 \mu \mathrm{g} / \mathrm{ml}$ metformin (concentration in therapeutical range) were also studied in a few experiments.

Gene expression studies Messenger RNA expression of beclin-1, ATG1 (also known as ULK1), LAMP2 and cathep$\sin \mathrm{B}, \mathrm{D}, \mathrm{F}$ and $\mathrm{H}$ was measured in islets by quantitative RT-PCR [2]. ATGs, lysosome-associated membrane proteins (LAMP) and cathepsins are involved in several phases of autophagy, from early steps to late degradation events $[2,4]$. Oligonucleotides were obtained from Assay-onDemand Gene Expression Products (Applied Biosystems, Foster City, CA, USA). Messenger RNA level was quantified (cycle thresholds were below 40) and normalised for beta actin [9].

Statistical analysis Data are expressed as mean $\pm \mathrm{SD}, n$ is the number of pancreases per given experimental setting. Differences were analysed by the two-tailed Student's $t$ test for unpaired data or ANOVA, as appropriate.

\section{Results}

Altered autophagy in diabetic beta cells In pancreatic samples, 4,112 endocrine cells were studied by electron microscopy. Dead beta cells accounted for $2.24 \pm 0.53 \%$ and $0.66 \pm 0.52 \%$ of beta cells in diabetic and control specimens, respectively $(p<0.01)$. Compared with normal beta cells (Fig. 1a), dead beta cells with signs of apoptotic death (Fig. 1b) made up $1.06 \pm 0.43 \%$ of beta cells in diabetic and $0.30 \pm 0.32 \%$ in control islets $(p<0.05)$. Dead beta cells with 


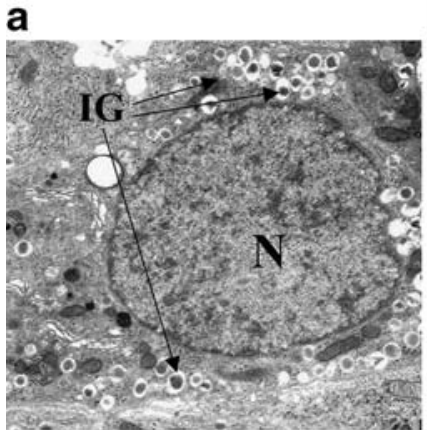

b

c

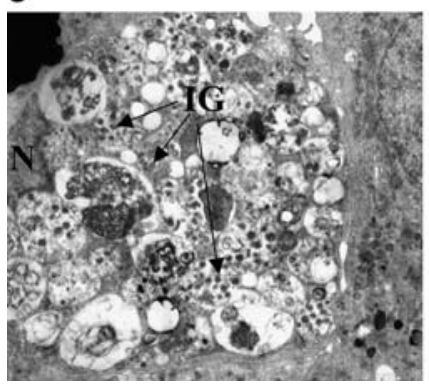

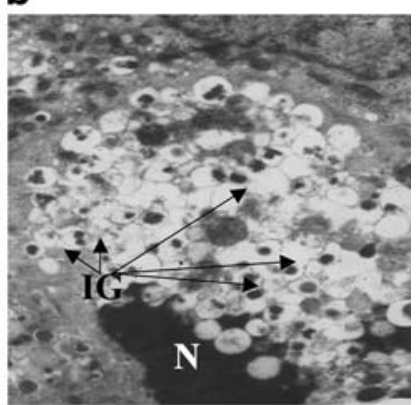

d

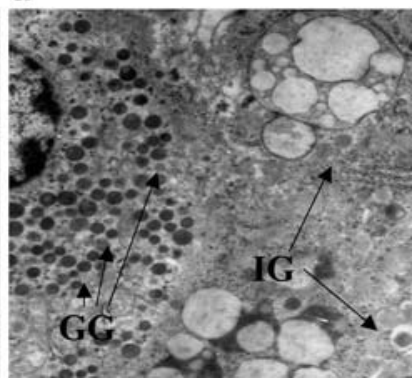

Fig. 1 Micrographs of a normal beta cell (a), an apoptotic type 2 diabetic beta cell with marked chromatin condensation (b), a dead type 2 diabetic beta cell with massive vacuole engulfment (c) and a type 2 diabetic alpha cell with no signs of altered autophagy, close to a beta cell with vacuolisation (d). GG, glucagon granules; IG, insulin granules; N, nucleus. Magnification: $\times 10,000(\mathbf{a}, \mathbf{c}), \times 7,000(\mathbf{b}, \mathbf{d})$

massive vacuole overload and no major chromatin condensation (signs of autophagy-associated cell death) (Fig. 1c) accounted for $1.18 \pm 0.54 \%$ of beta cells in diabetic and $0.36 \pm 0.26 \%$ in control islets $(p<0.05)$. Alpha cells (Fig. 1d) and delta cells (not shown) were not affected by autophagy alterations. Density volume $(\mathrm{ml} \%)$ of autophagic vacuoles and autophagosomes was higher $(0.62 \pm 0.11$ vs $0.11 \pm 0.05$ and $7.6 \pm 1.0$ vs $4.5 \pm 0.3$ respectively; $p<0.01)$ in diabetic beta cells.

Beclin-1 and ATG1 were similarly expressed in control and type 2 diabetes islets (Fig. 2). However, diabetic islets had significantly lower expression of $L A M P 2$ and cathepsin B and D, (Fig. 2). No difference was observed for cathepsin $\mathrm{F}$ and $\mathrm{H}$ (not shown).

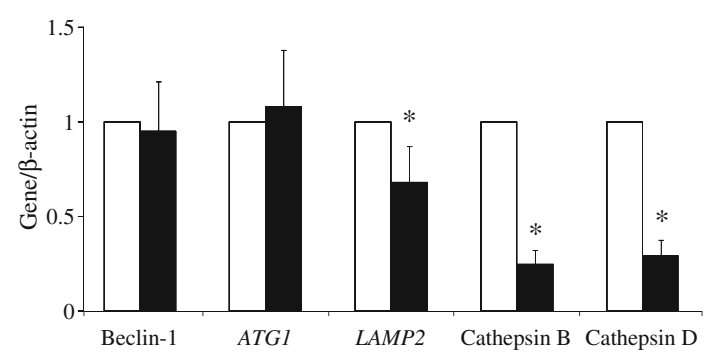

Fig. 2 Gene expression (ratio over $\beta$ actin) of beclin-1, ATG1, $L A M P 2$, cathepsin $\mathrm{B}$ and cathepsin $\mathrm{D}$ in control (white bars) and type 2 diabetic (black bars) islets. Islet preparations: $n=4-5 ;{ }^{*} p<0.05$ vs control (two-tailed Student's $t$ test)

Role of NEFA and metformin In non-diabetic islets, exposure to NEFA $(n=6)$ caused dead beta cells with vacuole engulfment to increase (from $0.9 \pm 0.3$ to $1.6 \pm 0.2 \%$ ), with improvement when metformin was added to NEFA-containing medium $(0.8 \pm 0.3 \%, p<0.05$ by ANOVA). Similar results were obtained with density volume of autophagic vacuoles $(0.4 \pm 0.1,1.1 \pm 0.2$ and $0.3 \pm 0.2 \mathrm{ml} \%$ respectively, $p<0.05$ by Bonferroni correction) and autophagosomes $(4.8 \pm 1.8,7.9 \pm$ 0.8 and $4.8 \pm 0.5 \mathrm{ml} \%$ respectively, $p<0.05$ by Bonferroni correction) (Fig. 3).

In diabetic islets, dead beta cells with vacuole overload decreased from $1.4 \pm 0.4$ to $0.6 \pm 0.3 \%(n=4, p<0.05)$ after metformin exposure. This was associated with reduction of autophagic vacuoles (from $0.4 \pm 0.1$ to $0.1 \pm 0.09 \mathrm{ml} \%$, $p<0.01$ ) and autophagosomes (from $7.3 \pm 1.1$ to $4.7 \pm$ $0.7 \mathrm{ml} \%, p<0.05$ ).

In addition, NEFA-exposed cells showed reduced $L A M P 2$ expression $(-57 \pm 15 \%, p<0.05)$. Both in diabetic and NEFA-exposed islets, metformin normalised LAMP2 transcription (not shown).

\section{Discussion}

Accumulation of autophagic vacuoles and autophagosomes in human type 2 diabetic beta cells, as well as increased levels of dead beta cells with vacuole engulfment as reported in this study are novel findings that may contribute to beta cell dysfunction in diabetes. Autophagic structures overload might be due to increased production and/or reduced removal. We found that expression of beclin-1 and ATG1, which are involved in early stages of autophagy, did not differ between control and diabetic islets. However, the reduced expression of LAMP2 and cathepsin B and D suggests alterations at later phases of autophagy (autophagosome maturation and lysosomal events) in diabetic beta cells. Similar findings have been reported in $\mathrm{Rab3a}^{-/-}$mice, where vacuole accumulation was observed, with several ATG genes not differently expressed and LAMP2 downregulated [6]. These observa- a

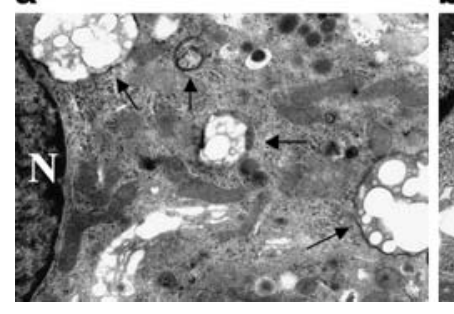

b

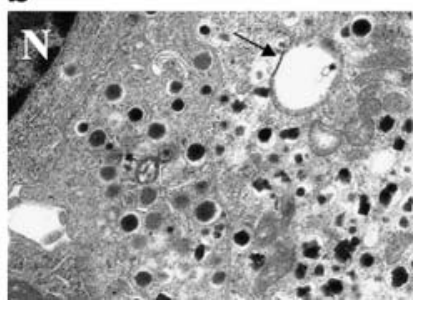

Fig. 3 Micrographs of beta cells from isolated islets exposed in vitro to NEFA without metformin (a) or to NEFA with metformin (b). Vacuolisation (arrows) is much more marked in beta cells exposed to NEFA without metformin (see text for quantification). Magnification: $\times 10,000$. N, nucleus 
tions suggest that hampered removal may lead to accumulation of autophagic material in type 2 diabetes beta cells. However, it should be kept in mind that cathepsins can also be found in intracellular organelles other than lysosomes [11].

Massive engulfment with autophagic vacuoles characterises the form of programmed cell death that is called autophagic cell death [2]. We found increased amounts of dead beta cells with signs of altered autophagy in samples from type 2 diabetic patients. Although it is still unclear whether autophagy-mediated death is an entity in itself or part of other means of cell death, some evidence suggests that the autophagic process may be a prerequisite for cell death in conditions where apoptosis is inhibited [12]. Currently, there is little doubt that apoptosis is a major beta cell death mechanism in type 2 diabetes [1, 9]. Nevertheless, our results highlight the possibility that targeting apoptosis might potentially induce non-apoptotic cell death, including autophagy.

Similar to results reported elsewhere [3], we observed that exposure to NEFA generated marked vacuole accumulation and increased beta cell death. This might be due to NEFA-induced ceramide formation, which, in turn, can promote intracellular accumulation of autophagic vacuoles [13]. In our study, this was associated with reduced LAMP2 expression, again suggesting alterations at the level of autophagic structures removal.

The present study also shows that metformin can reduce autophagic vacuole accumulation and autophagic beta cell death both in beta cells from type 2 diabetic patients and in NEFA-exposed beta cells from non-diabetic controls, a finding associated with restored $L A M P 2$ expression. At the present time we can only speculate on the mechanisms leading to these effects. Metformin potentiates AMP kinase (AMPK) activity [14]. Beside several other actions, AMPK can inhibit the mammalian target of rapamycin (mTOR) [15]. Since mTOR inhibition leads to increased removal of autophagic material [15], it is possible that metformin promotes elimination of autophagic vacuoles by reducing the action of mTOR on autophagy.

Whereas more work is needed to understand the precise role of autophagy and its regulation in diabetes, the present results show that pancreatic beta cells in human type 2 diabetes have signs of altered autophagy, associated with a form of beta cell death that is morphologically distinct from apoptosis and probably due to altered removal of autophagic structures. These alterations were reproduced by exposure to NEFA and could benefit from pharmacological intervention. On a more general note, we suggest that therapeutical strategies directed at multiple cell-death path- ways may be required to preserve beta cell mass in type 2 diabetes.

Acknowledgements This work was supported in part by the Italian Ministry of University and Research (PRIN 2007-2008) and the European Community (LSHM-CT-2006-518153).

Duality of interest The authors declare that there is no duality of interest associated with this manuscript.

\section{References}

1. Meier JJ (2008) Beta cell mass in diabetes: a realistic therapeutic target? Diabetologia 51:703-713

2. Galluzzi L, Maiuri MC, Vitale I et al (2007) Cell death modalities: classification and pathophysiological implications. Cell Death Differ 14:1237-1243

3. Ebato C, Uchida T, Arakawa M et al (2008) Autophagy is important in islet homeostasis and compensatory increase of beta cell mass in response to high-fat diet. Cell Metab 8:325-332

4. Eskelinen EL, Saftig P (2009) Autophagy: a lysosomal degradation pathway with a central role in health and disease. Biochim Biophys Acta doi:10.1016/j.bbamcr.2008.07.014

5. Mandrup-Poulsen T, Egeberg J, Nerup J, Bendtzen K, Nielsen JH, Dinarello CA (1987) Ultrastructural studies of time-course and cellular specificity of interleukin-1 mediated islet cytotoxicity. Acta Pathol Microbiol Immunol Scand 95:55-63

6. Kaniuk NA, Kiraly M, Bates H, Vranic M, Volchuk A, Brumell $\mathrm{JH}$ (2007) Ubiquinated-protein aggregates form in pancreatic beta-cells during diabetes-induced oxidative stress and are regulated by autophagy. Diabetes 56:930-939

7. Marsh BJ, Soden C, Alarcon C et al (2007) Regulated autophagy controls hormone content in secretory-deficient pancreatic endocrine beta-cells. Mol Endocrinol 21:2255-2269

8. Jung HS, Chung KW, Won Kim J et al (2008) Loss of autophagy diminishes pancreatic beta cell mass and function with resultant hyperglycemia. Cell Metab 8:318-324

9. Marchetti P, Bugliani M, Lupi R et al (2007) The endoplasmic reticulum in pancreatic beta cells of type 2 diabetes patients. Diabetologia 50:2486-2494

10. Lupi R, Dotta F, Marselli L et al (2002) Prolonged exposure to free fatty acids has cytostatic and pro-apoptotic effects on human pancreatic islets: evidence that beta-cell death is caspase mediated, partially dependent on ceramide pathway, and Bcl-2 regulated. Diabetes 51:1437-1442

11. Hook VY (2006) Unique neuronal functions of cathepsin L and cathepsin B in secretory vesicles: biosynthesis of peptides in neurotransmission and neurodegenerative disease. Biol Chem 387:1429-1439

12. Gozuacik D, Kimchi A (2004) Autophagy as a cell death and tumor suppressor mechanism. Oncogene 23:2891-2906

13. Scarlatti F, Bauvy C, Ventruti A et al (2004) Ceramide-mediated macroautophagy involves inhibition of protein kinase $\mathrm{B}$ and up-regulation of beclin 1. J Biol Chem 279:18384-18391

14. Rutter GA, Leclerc I (2009) The AMP-regulated kinase family: enigmatic targets for diabetes therapy. Mol Cell Endocrinol 297:41-49

15. Meijer AJ, Codogno P (2007) AMP-activated protein kinase and autophagy. Autophagy 3:238-240 